\title{
Improved wait time intervals for prostate cancer patients in a multidisciplinary rapid diagnostic unit compared to a community- based referral pattern
}

\author{
Perakaa Sethukavalan; ${ }^{*}$ Liying Zhang, MD, PhD; ${ }^{;}$Vibhuti Jethava; ${ }^{*}$ Christiaan Stevens, MD, FRCPC; ${ }^{\dagger}$ Stanley \\ Flax, MD, FRCSC,; Roger Buckley, MD, FRCSC,; Susan Bondy, PhD;: Andrew Loblaw, MD, MSc, FRCPC*
}

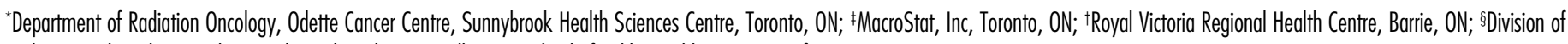
Urology, North York General Hospital, North York, ON; *Dalla Lana School of Public Health, University of Toronto, Toronto, ON

See related article on page 251 .

Cite as: Can Urol Assoc J 2013;7(7-8):244-50. http://dx.doi.org/10.5489/cuaj.181

Published online August 19, 2013.

\section{Abstract}

Background: Wait times in cancer diagnosis and treatment may significantly affect a patient's treatment outcome, prognosis and quality of life. The purpose of this study was to capture wait time intervals for patients with prostate cancer treated with radiotherapy (RT) at the Odette Cancer Centre, Toronto, Ontario, Canada and to compare patients diagnosed in a rapid diagnostic unit (RDU) versus the usual community referral process.

Methods: Patients agreed to participate in the study during their RT planning sessions. A semi-structured interview and chart abstraction was conducted to record key wait time milestones.

Results: A total of 87 patients participated in the study: 44 RDU patients and 43 community patients. The median overall wait time intervals from suspicion of prostate cancer to RT was 138 and 183 days, respectively $(p=0.046)$. There were statistically significant differences observed for other key wait time intervals favouring the RDU cohort: suspicion to decision-to-treat (DTT; $p=0.012)$, urologist visit to diagnosis $(p=0.0094)$, diagnosis to DTT ( $p=0.018)$, and diagnosis to treatment $(p=0.016)$. Risk category and Gleason sum was independently predictive of longer intervals from diagnosis to DTT.

Interpretation: Wait time intervals from suspicion to treatment are significantly shorter for prostate cancer patients in 2011 to 2012 than in 2003 when patients were diagnosed and referred in the community setting. A prostate-specific RDU further reduced a number of key wait time intervals supporting more multidisciplinary RDUs for common diseases. Further work needs to be done to identify why delays are occurring and to develop new processes to minimize delays.

\section{Introduction}

Wait times in cancer diagnosis and treatment are important measures of how quickly patients are getting access to care. Prolonged wait times may significantly affect a patient's treatment outcomes, prognosis and quality of life. ${ }^{1}$ While some wait times are reasonable and required to plan treatment, long delays may cause significant emotional distress for patients and their caregivers. ${ }^{1}$ In Ontario, for the fiscal year 2002-2003, the median wait time for radiation therapy was 44 days from time of patient referral to radiotherapy (RT) treatment. ${ }^{2}$ Our group previously audited this prostate cancer diagnosis and treatment pathway at the Odette Cancer Centre, Toronto, Ontario, Canada. The median interval from suspicion of prostate cancer to diagnosis was 53 days, while the median wait time from diagnosis to treatment was 127 days in the usual community diagnostic and referral process. ${ }^{3}$ In November 2004, Ontario's Ministry of Health and Long Term Care announced a wait time strategy to reduce wait times and improve access to care, including cancer treatment. As part of the initiative, Cancer Care Ontario started reporting wait times across different cancer sites. Since December 2005, the provincial target for wait times for RT is within 28 days of the patient being ready to treat. ${ }^{4}$ With the increasing number of anticipated prostate cancer cases being diagnosed, it is important to identify delays within the diagnosis and treatment pathway. ${ }^{5}$

In 2007, a rapid diagnostic unit (RDU) called The Gale and Graham Wright Prostate Centre was set up at the North York General Hospital (NYGH), Branson Site, Toronto. The Wright Prostate Centre is a diagnostic assessment program and multidisciplinary clinic where both radiation oncology and urology specialists see every patient on the same day after obtaining their biopsy results. It was hypothesized that, compared to the usual community diagnostic and referral process, the RDU would reduce wait times from suspicion 
of prostate cancer to decision-to-treat (DTT). Our clinical impression was that patients were diagnosed and treated faster through this route compared to those referrals through the usual channels of care.

The purpose of this study was to document intervals between key wait time milestones within the prostate cancer care pathway from suspicion to the start of definitive therapy for patients referred to and treated with radical RT at the Odette Cancer Centre, comparing patients diagnosed in the RDU versus the usual community process.

\section{Methods}

Research ethics board approval (REB \#067-2011) was obtained prior to opening the study. Patients were screened when they came in for planning for radiation with curative intent for their newly diagnosed, biopsy-confirmed prostate adenocarcinoma. Written consent was obtained for all patients. Patients initially managed on active surveillance, those who were too unwell to participate or patients of physicians who declined to participate in the study were excluded.

We accrued a convenience sample of 100 consenting patients ( 50 from the community and 50 from the RDU), who were treated with radical RT at the Odette Cancer Centre at Sunnybrook Health Sciences Centre, in 2011 and 2012. The sample was obtained at Odette, and not at the $R D U$, because the RDU is not equipped to treat patients with RT. All patients who are seen at the RDU were referred to Odette for treatment. The first 50 patients who were seen at the RDU and the first 50 patients from the community who consented were accrued to the study on a consecutive basis starting in October 2011. The number of patients who declined to participate in the study was not recorded.

\section{Data collection methods}

A semi-structured interview was conducted to measure wait time intervals in the prostate cancer care pathway. Patients were questioned regarding basic demographic information, such as marital status, language spoken in the home and level of education.

\section{Key wait time milestones in the care pathway}

The best estimation of key wait time milestones in the care pathway were recorded and verified through medical chart abstraction by trained personnel. Time points, such as date of first suspicion, date of urologist referral, date of diagnosis (i.e., date when biopsy was preformed), date of radiation oncologist consult, dates of staging tests, dates of radiation simulation, and first date of radiation therapy (or hormonal treatment) were recorded (Table 1). Time data were extracted from interviews for the purpose of identifying key dates not recorded in the hospital charts, such as date of first suspicion.

Patients were also questioned whether they perceived delay in the care pathway. If a delay was perceived, they were asked to categorize the type of delay (systematic, patient-initiated, physician-related or other).

\section{Statistical analyses}

Simple descriptive analysis was conducted for all patients and for those in the two different cohorts. Results were expressed as median and ranges for age; Gleason score and prostate-specific antigen (PSA) at pre-treatment; proportions for categorical variables, such as age (>70 vs. $\leq 70)$, Gleason sum, ${ }^{6-9}$ clinical T-Stage (T1-T2b, T2c, T3a), pre-treatment PSA ( $\geq 10$ vs. $<10$ ), risk stratification (low, intermediate, or high) and treatment delayed or not. Risk stratification was defined as per the Canadian Genitourinary Radiation Oncology Consensus and National Comprehensive Cancer Network. ${ }^{6,7}$

To compare demographics between the two cohorts, we conducted the Fisher exact test (for categorical variables) or Wilcoxon rank-sum test (for continuous variable). To compare wait time intervals between the two cohorts, we performed a one-way analysis of variance (ANOVA). Natural logarithm transformation was conducted for all wait time

\begin{tabular}{|c|c|}
\hline Term & Definition \\
\hline Suspicion & $\begin{array}{l}\text { The date of first suspicion was defined as either the date on which the family physician first became suspicious } \\
\text { based on a PSA test, when the physician or patient became suspicious because of symptoms, or an abnormal DRE, } \\
\text { which warranted further testing or referral to a specialist to definitively diagnose or rule out cancer. }{ }^{1}\end{array}$ \\
\hline Urologist referral & The date the patient was seen by a urologist for suspicion of prostate cancer. \\
\hline Diagnosis & The date the biopsy was performed. \\
\hline RO consult & The date the patient was seen by a RO regarding potential radiotherapy treatment. \\
\hline Decision to treat & $\begin{array}{l}\text { The date the physician entered the order for radiotherapy into eSheet, the electronic ordering system at the Odette } \\
\text { Cancer Centre. }\end{array}$ \\
\hline RT & $\begin{array}{l}\text { The first treatment is the date on which the first therapeutic service or procedure is performed, whether it is the } \\
\text { first day of RT or the date of the first hormone injection. }{ }^{1}\end{array}$ \\
\hline
\end{tabular}


Sethukavalan et al.

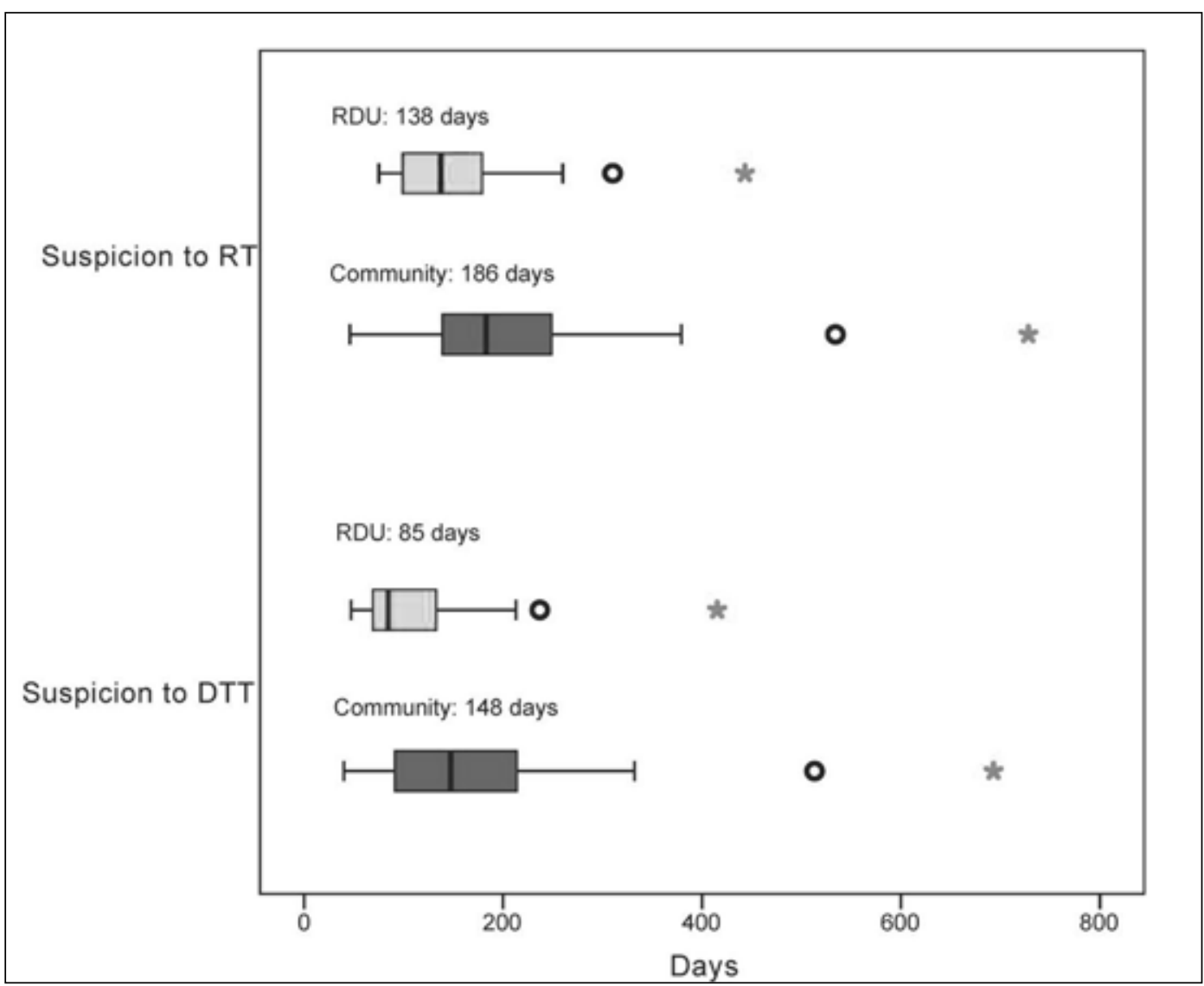

Fig. 1. Box and whisker plot of wait time intervals, in days, from suspicion to radiotherapy and from suspicion to decision-totreat. RDU: rapid diagnostic unit; RT: radiation therapy; DTT: decision-to-treat.

intervals to normalize the distribution. The outcome was each wait time interval (log scale) in days, and the independent variable was a binary variable of cohort $(1=$ community, $0=\mathrm{RDU}$ ). The coefficient and standard error (SE) of the cohort variable, and the mean squared error (MSE) was estimated for each model. A positive coefficient indicates that the community cohort had longer days than the RDU cohort. The MSE was estimated for the error variance, with a lower MSE indicating better fit. To search for significant demographic factors related to each time interval, two-way ANOVA was performed after adjusting for cohorts. The independent variables in the two-way ANOVA were binary variables of cohort and demographic categorical variables (such as age, Gleason score, clinical T-stage, pre-treatment PSA, or risk stratification). The interaction between cohort and demographics were also tested in the model. $P$ values less than 0.05 were considered statistically significant. Box and whisker plots of wait time intervals were performed for patients in the two cohorts. All analysis was conducted by Statistical Analysis Software (SAS v. 9.2 for Windows).

\section{Results}

The study aimed to include 100 patients. Of the 51 consenting RDU patients, 7 were excluded because they were on active surveillance prior to treatment. Of the 53 consenting community patients, 10 were excluded because they were on active surveillance $(n=8)$ or were seen initially at another radiotherapy centre $(n=2)$. In total, the study included 87 patients (44 RDU and 43 community patients).

When the RDU cohort was stratified by risk according to PSA, Gleason sum, and T-category, 14 patients (32\%) had high-risk, 18 patients (41\%) intermediate-risk and 12 patients $(27 \%)$ low-risk compared to the community cohort in which 14 patients (33\%) had high-risk, 23 patients (53\%) intermediate-risk, and 6 patients (14\%) low-risk.

Table 2 shows patients' characteristics overall and in the two cohorts. There were no significant differences between the RDU and community cohorts with respect to demographic (not all data shown) or tumour variables. 


\begin{tabular}{|c|c|c|c|c|}
\hline & $\begin{array}{c}\text { All patients } \\
(n=87)\end{array}$ & $\begin{array}{c}\text { RDU } \\
(n=44)\end{array}$ & $\begin{array}{c}\text { Community } \\
(n=43)\end{array}$ & $\begin{array}{c}p \\
\text { value* }\end{array}$ \\
\hline $\begin{array}{l}\text { Median age } \\
\text { (range) }\end{array}$ & $70(48-84)$ & $68(49-84)$ & $72(48-83)$ & 0.22 \\
\hline $\begin{array}{l}\text { Median Gleason } \\
\text { score (range) }\end{array}$ & $7(6-9)$ & $7(6-9)$ & $7(6-9)$ & 0.50 \\
\hline \multirow{2}{*}{$\begin{array}{l}\text { Median pre- } \\
\text { treatment PSA } \\
\text { (range) }\end{array}$} & $7(0-248)$ & $7(0-248)$ & $8(3-174)$ & 0.10 \\
\hline & n (\%) & $\mathrm{n}(\%)$ & n (\%) & \\
\hline Age & & & & 0.29 \\
\hline$\leq 70$ & $44(50.6 \%)$ & $25(56.8 \%)$ & $19(44.2 \%)$ & \\
\hline$>70$ & $43(49.4 \%)$ & $19(43.2 \%)$ & $24(55.8 \%)$ & \\
\hline Gleason score & & & & 0.18 \\
\hline 6 & $20(23.0 \%)$ & $13(29.6 \%)$ & $7(16.2 \%)$ & \\
\hline 7 & $54(62.0 \%)$ & $23(52.2 \%)$ & $31(72.1 \%)$ & \\
\hline $8-9$ & $13(15.0 \%)$ & $8(18.2 \%)$ & $5(11.6 \%)$ & \\
\hline T-stage & & & & 0.63 \\
\hline T1-T2b & $79(90.8 \%)$ & $41(93.2 \%)$ & $38(88.4 \%)$ & \\
\hline $\mathrm{T} 2 \mathrm{c}$ & $3(3.4 \%)$ & $1(2.3 \%)$ & $2(4.7 \%)$ & \\
\hline T3a & $5(5.8 \%)$ & $2(4.5 \%)$ & $3(7.0 \%)$ & \\
\hline $\begin{array}{l}\text { PSA at pre- } \\
\text { treatment }\end{array}$ & & & & 0.11 \\
\hline$<10$ & $58(66.7 \%)$ & $33(75.0 \%)$ & $25(58.1 \%)$ & \\
\hline$\geq 10$ & $29(33.3 \%)$ & $11(25.0 \%)$ & $18(41.9 \%)$ & \\
\hline Risk stratification & & & & 0.29 \\
\hline High & $28(32.2 \%)$ & $14(31.8 \%)$ & $14(32.6 \%)$ & \\
\hline Intermediate & $41(47.1 \%)$ & $18(40.9 \%)$ & $23(53.4 \%)$ & \\
\hline Low & $18(20.7 \%)$ & $12(27.3 \%)$ & $6(14.0 \%)$ & \\
\hline
\end{tabular}

\section{Wait time intervals}

The median overall wait time interval from suspicion of prostate cancer to RT, the "overall interval," was 138 days for the RDU cohort and 183 days for the community cohort $(p=0.046)$. The median wait time interval from suspicion of prostate cancer to diagnosis, the "diagnostic interval," for patients in the RDU and community cohorts was 49 days and 67 days, respectively $(p=0.29)$. The median wait time interval from diagnosis to radiation oncology $(\mathrm{RO})$ consult for patients in the RDU and community cohorts was 27 days and 49 days, respectively $(p=0.0019)$. The median wait time interval from RO consult to start of therapy (RT), the "treatment interval," for patients in the RDU and community cohorts was 46 days and 37 days, respectively $(p=0.52)$ (Fig. 1).

The box plot indicates wait time intervals in days for patients from the RDU (green) or community (blue) site. The boxes represents values between the 25 th and 75 th percentiles; the midline marks the median; the whiskers (error bars), the most extreme values in the data set that were not more than 1.5 times the width of the box (interquartile range [IQR]) beyond either quartile; open circles represent outliers (1.5-3.0 times the IQR); and red stars represent extremes (>3.0 times the IQR) (Fig. 1).

There were statistically significant differences between the two cohorts, favouring the RDU cohort, for other key wait time intervals (Fig. 2). These differences included suspicion to DTT ( $p=0.012)$, urologist visit to diagnosis $(p=0.0094)$, diagnosis to DTT $(p=0.018)$, and diagnosis to treatment $(p=0.016)$ (Table 3$)$.

Wait time intervals identified by grey arrows were significantly lower in the RDU cohort compared to the community cohort (non-significant differences identified by black arrows).

If we assume to have pooled standard deviations between the two groups, group sample sizes of 44 and 43 can achieve $81 \%$ power to detect a difference between 138 days and 183 days, with estimated group standard deviations of 90, with a significance level of 0.05 using a two-sided MannWhitney test. As there were some significant differences on some wait time intervals between the two cohorts, significant relationships between demographics and interval lengths were sought, adjusting for cohort as a confounding factor.

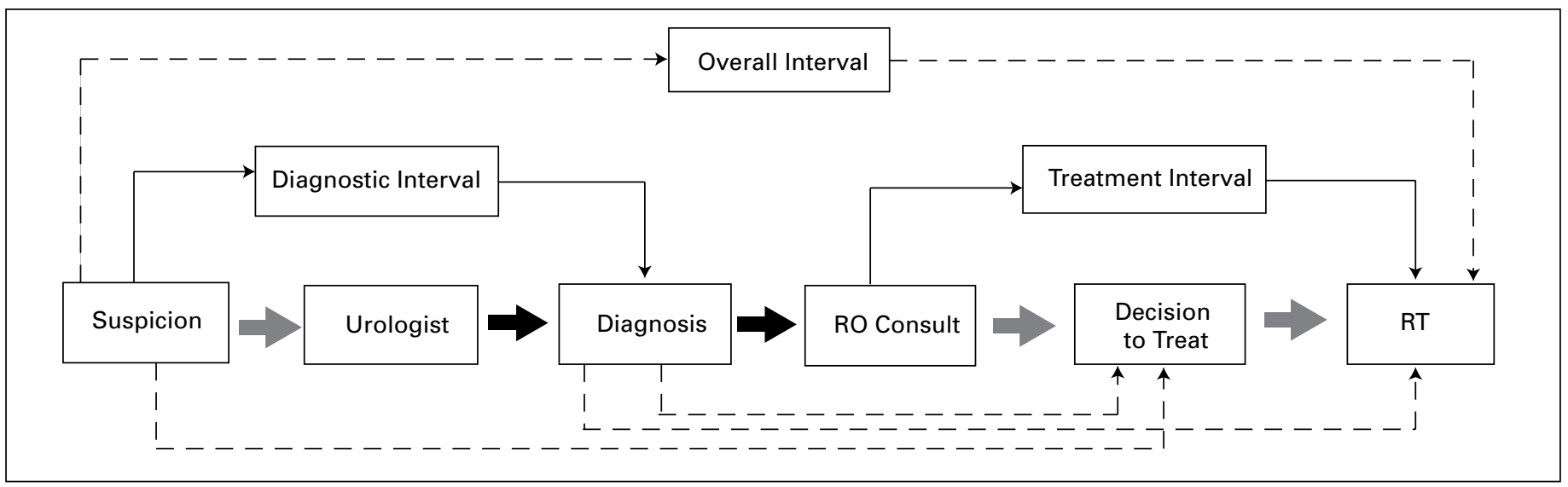

Fig. 2. Timeline of wait time intervals in the prostate cancer care pathway. RO: radiation oncology; RT: radiation therapy. 
Sethukavalan et al.

\begin{tabular}{|c|c|c|c|c|c|}
\hline & All & & RDU (n=44) & Community ( $n=43$ ) & $p$ value* \\
\hline \multicolumn{6}{|c|}{ Treatment delayed } \\
\hline No & 65 & $(74.71 \%)$ & $37(84.09 \%)$ & $28(65.12 \%)$ & 0.051 \\
\hline Yes & 22 & $(25.29 \%)$ & $7(15.91 \%)$ & $15(34.88 \%)$ & \\
\hline \multicolumn{6}{|c|}{ Type of delay (when treatment delayed=Yes) } \\
\hline Systematic & 14 & (63.64\%) & $4(57.14 \%)$ & $10(66.67 \%)$ & 86 \\
\hline Patient & 3 & $(13.64 \%)$ & $1(14.29 \%)$ & $2(13.33 \%)$ & \\
\hline Physician & 1 & $(4.55 \%)$ & $0(0.00 \%)$ & $1(6.67 \%)$ & \\
\hline Other & 4 & $(18.18 \%)$ & $2(28.57 \%)$ & $2(13.33 \%)$ & \\
\hline
\end{tabular}

After adjustment, demographics were not predictive of any of the wait time intervals (data not shown).

Risk category and Gleason sum were independently predictive of longer intervals from diagnosis to DTT. Patients with Gleason sum of 6 or 7 disease had shorter diagnosis to DTT intervals compared patients with Gleason 8-9 disease (median 41 vs. 42 vs. 94 days, respectively, $p=0.035$ ). Patients with low- versus intermediate- versus low-risk disease had median intervals of 67 vs. 41 vs. 38 days, respectively ( $p=0.011)$. Conversely, patients with PSA $\geq 10$, or with high-risk disease had shorter DTT to RT intervals: PSA $\geq 10$ vs. $<10: 22$ vs. 36 days, $p=0.049$; high- versus favourablerisk: 21 vs. 40 days $(p=0.0056)$.

\section{Patient perceived delays}

Among all patients, there were 22 patients (25\%) with perceived delayed treatment. Among those 22 patients, most of patients identified a systematic reason (64\%) for the delay (Table 4). There was no significant difference on the cause of perceived delay between the RDU and community cohorts $(p=0.86)$.

\section{Discussion}

In this study, wait time intervals along the entire trajectory of the prostate cancer care pathway were recorded for patients receiving RT with curative intent. Our main objective was to determine whether wait time intervals were shorter for patients diagnosed and referred through the Wright Prostate Centre (a prostate-specific, multidisciplinary RDU) compared to the usual community process. To our knowledge, the only other study published that had documented wait time intervals from suspicion of cancer to treatment in this population was conducted by our group in 2003. ${ }^{3}$ However, a wait time study was conducted by Grunfeld and colleagues in Ottawa, Ontario on suspected lung, colorectal and prostate cancer patients. These authors collected wait time intervals prospectively from referral to diagnostic assessment to the first treatment. Patients who were identified to have prostate cancer had a mean wait time of 114 days. First treatment was defined as neoadjuvant chemotherapy, surgery date, chemo- therapy, radiotherapy or date of decision for no treatment. However, wait times were not reported based on treatment the patient received..$^{10}$

Our results from 2011-2012 suggest that the median overall wait time interval from suspicion of prostate cancer to the start of treatment in the community setting has shortened by over 2 months compared to 2003 (183 vs. 247 days). ${ }^{3}$ More impressive was that the same interval was 45 days shorter when patients were diagnosed and referred through the Wright Prostate Centre. We believe this is a key outcome as patients and their families say that waiting is one of the worst parts of the cancer journey. ${ }^{8}$

We observed that intervals from diagnosis to DTT were significantly longer (after adjusting for referral cohort) for patients with high-grade or high-risk disease. However, this observation is likely explained by additional wait times introduced by organizing staging investigations (not routinely done for patients with low- and intermediate-risk disease). ${ }^{9}$ However, the time to DTT to the start of treatment intervals were shorter for high-risk patients - this time likely reflects a shorter delay in initiating androgen deprivation therapy (standard of care with RT for high-risk disease ${ }^{11}$ ) than RT.

The median wait time interval from RO consult to the start of treatment was 42 and 49 days for the RDU and community cohorts, respectively, but both were well over the recommended wait time of 28 days from the patient being ready to treat. ${ }^{4}$ This has important implications for future healthcare delivery to this population. While most prostate cancers that are diagnosed are not destined to be fatal, the earlier diagnosis and treatment of prostate cancer will likely result in decreased anxiety; for high-grade disease, intervals from diagnosis to treatment longer than 2.5 months have been associated with worse outcomes. ${ }^{12}$ Of the 28 patients with high-risk disease in this series, $57 \%$ of the RDU patients and $29 \%$ of the community patients had treatment started within 2.5 months of diagnosis ( $p=0.25$, Fisher exact test). More work is needed to further reduce wait times to meet provincial and national wait time targets, particularly in high-risk disease.

PSA screening is very controversial. Guidelines from the US Task Force and American Society of Clinical Oncology (ASCO) offer conflicting recommendations for serial PSA 


\begin{tabular}{|c|c|c|c|c|}
\hline Interval lengths (days) & All patients $(n=87)$ & RDU (n=44) & Community ( $n=43$ ) & $p$ value* \\
\hline \multicolumn{5}{|l|}{ Overall interval } \\
\hline Suspicion to RT & & & & 0.046 \\
\hline Median (IQR) & $160(111-219)$ & $138(99-179)$ & $183(139-249)$ & \\
\hline Mean (95\% Cl) & $188(158-218)$ & $158(128-187)$ & $218(166-270)$ & \\
\hline \multicolumn{5}{|l|}{ Diagnostic interval } \\
\hline Suspicion to diagnosis & & & & 0.29 \\
\hline Median (IQR) & $53(37-95)$ & $49(35-90)$ & $67(45-102)$ & \\
\hline Mean $(95 \% \mathrm{Cl})$ & $94(65-123)$ & $74(48-99)$ & $114(62-167)$ & \\
\hline Suspicion to urologist & & & & 0.86 \\
\hline Median (IQR) & $29(17-47)$ & $30(17-46)$ & $26(17-47)$ & \\
\hline Mean $(95 \% \mathrm{Cl})$ & $34(26-43)$ & $35(22-48)$ & $33(21-45)$ & \\
\hline Urologist to diagnosis & & & & 0.0094 \\
\hline Median (IQR) & $23(14-56)$ & $20(9-33)$ & $39(15-90)$ & \\
\hline Mean $(95 \% \mathrm{Cl})$ & $65(36-93)$ & $29(18-40)$ & $100(46-153)$ & \\
\hline \multicolumn{5}{|l|}{ Consult interval } \\
\hline Diagnosis to RO & & & & 0.0019 \\
\hline Median (IQR) & $33(23-53)$ & $27(20-34)$ & $49(28-79)$ & \\
\hline Mean $(95 \% \mathrm{Cl})$ & $45(36-54)$ & $32(24-39)$ & $59(43-74)$ & \\
\hline \multicolumn{5}{|l|}{ Treatment interval } \\
\hline $\mathrm{RO}$ to $\mathrm{RT}$ & & & & 0.52 \\
\hline Median (IQR) & $42(27-83)$ & $46(28-76)$ & $37(22-89)$ & \\
\hline Mean $(95 \% \mathrm{Cl})$ & $61(50-71)$ & $60(47-72)$ & $62(45-78)$ & \\
\hline RO to decision-to-treat & & & & 0.72 \\
\hline Median (IQR) & $2(0-21)$ & $4(1-20)$ & $2(0-24)$ & \\
\hline Mean $(95 \% \mathrm{Cl})$ & $22(12-31)$ & $21(9-34)$ & $23(8-37)$ & \\
\hline Decision-to-treat to RT & & & & 0.94 \\
\hline Median (IQR) & $34(21-62)$ & $40(27-58)$ & $31(20-76)$ & \\
\hline Mean $(95 \% \mathrm{Cl})$ & $46(38-53)$ & $42(35-49)$ & $49(35-63)$ & \\
\hline \multicolumn{5}{|l|}{ Other intervals } \\
\hline Suspicion to decision-to-treat & & & & 0.012 \\
\hline Median (IQR) & $117(78-182)$ & $85(69-133)$ & $148(91-214)$ & \\
\hline Mean $(95 \% \mathrm{Cl})$ & $151(121-181)$ & $117(88-145)$ & $185(133-237)$ & \\
\hline Diagnosis to decision-to-treat & & & & 0.018 \\
\hline Median (IQR) & $45(28-83)$ & $34(27-59)$ & $64(34-99)$ & \\
\hline Mean $(95 \% \mathrm{Cl})$ & $67(54-80)$ & $53(39-67)$ & $81(60-102)$ & \\
\hline Diagnosis to RT & & & & 0.016 \\
\hline Median (IQR) & $89(60-144)$ & $79(55-111)$ & $112(71-158)$ & \\
\hline Mean $(95 \% \mathrm{Cl})$ & $105(93-118)$ & $91(76-106)$ & $120(101-139)$ & \\
\hline
\end{tabular}

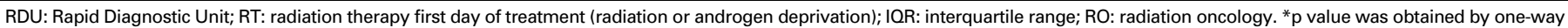
ANOVA.

screening. Several randomized control studies have been conducted with conflicting results and have found no survival benefit. However, without PSA screening, it may be extremely difficult to identify patients who are high risk. The reason a convenience sample of 100 people was chosen was because this is a pilot project done at a single centre. The study was conducted at 1 centre for convenience - a stepping stone to setting up a bigger study to assess wait times across multiple institutions.

There are limitations to the study. The study was conducted retrospectively and some of the information was based on the patient's ability to recall dates, such as the date of suspicion and urologist referral and was only confirmed if the patient's chart contained transcription notes from the referring urologist or family physician. In addition, the study was conducted with a modest sample size over a limited time frame and, therefore, may not reflect population-based wait time intervals in the prostate cancer care pathway. Other variables, such as missed or cancelled appointments that could influence overall wait times, could not be assessed and may have significantly contributed to wait times. For example in our previous study, ${ }^{3}$ we reported that $30 \%$ of the 
Sethukavalan et al.

delays were patient, not system driven; in this study it was $13 \%$. Future studies should incorporate methodologies to assess true systematic delays versus patient-driven delays.

\section{Conclusions}

Wait time intervals from suspicion to treatment are significantly shorter for prostate cancer patients in 2011-2012 than in 2003 when patients are diagnosed and referred in the community setting. A prostate-specific RDU further reduced a number of key wait time intervals supporting more multidisciplinary RDUs for common diseases. However, even in the RDU system, the minority of patients meet provincial consensus-based wait time standards. Further work needs to be done to identify why delays are occurring and develop new processes to minimize delays.

Acknowledgements: The authors would like to acknowledge Gale and Graham Wright and all donors for their generous contributions to the Gale and Graham Wright Prostate Centre. Partial funding for this study came from the CARO-Abbott Urologic Research Award (CARO-ACURA).

Competing interests: None declared.

This paper has been peer-reviewed.

\section{References}

1. Cancer Care Ontario. Understanding Wait Times. Toronto: Cancer Care Ontario; 2011. https://www. cancercare.on.ca/ocs/wait-times/understandingwt/. Accessed July 30, 2013.

2. Canadian Institute for Health Information. CIHI-(3Ia-DM) Median wait time for radiation therapy for prostate cancer for fiscal year 2002/2003. http://www.cihi.ca/cihi-ext-portal/internet/en/document/ health+system+performance/indicators/health/prtwg_dm_31a. Accessed July 30, 2013.

3. Stevens C, Bondy SJ, Loblaw DA. Wait times in prostate cancer diagnosis and radiation treatment. Can Urol Assoc J 2010;4:243-8. http://dx.doi.org/10.5489/cuai.09122

4. Ministry of Health and Long-Term Care. Ontario wait times: Wait time targets. http://www.health.gov. on.ca/en/pro/programs/waittimes/surgery/target.aspx. Accessed August 15, 2013.

5. Quon H, Loblaw DA, Nam R. Dramatic increase in prostate cancer cases by 2021. BJU Int 2011;108:17348. http://dx.doi.org/10.1111/j.1464-410X.2011.10197.x

6. Lukka H, Warde P, Pickles T, et al. Controversies in prostate cancer radiotherapy: consensus development. Can J Urol 2001;8:1314-22.

7. Mohler J, Bahnson RR, Boston B, et al. NCCN Clinical Practice Guidelines in Oncology: Prostate Cancer. J Natl Compr Canc Netw 2010;8:162-200.

8. Flory N, Lang EV. Distress in the radiology waiting room. Radiology 2011;260:166-73. http://dx.doi. org/10.1148/radiol.11102211

9. Danielson $B$, Brundage $M$, Pearcey $R$, et al. Development of indicators of the quality of radiotherapy for localized prostate cancer. Radiother Oncol 2011;99:29-36. http://dx.doi.org/10.1016/i. radonc.2011.02.013

10. Grunfeld $E$, Watters $J M$, Urquhart $R$, et al. A prospective study of peri-diagnostic and surgical wait times for patients with presumptive colorectal, lung, or prostate cancer. Br J Cancer 2009; 100:56-62. http:// dx.doi.org/10.1038/si.bj..6604819

11. Bolla M, Van Tienhoven $G$, Warde $P$, et al. External irradiation with or without long-term androgen suppression for prostate cancer with high metastatic risk: 10-year results of an EORTC randomised study. Lancet Oncol 2010;11:1066-73. hittp://dx.doi.org/10.1016/S1470-2045(10)70223-0

12. Nguyen PL, Whittington R, Koo $S$, et al. The impact of a delay in initiating radiation therapy on prostatespecific antigen outcome for patients with clinically localized prostate carcinoma. Cancer 2005; 103:20539. http://dx.doi.org/10.1002/cncr.21050

Correspondence: Dr. Andrew Loblaw, Department of Radiation Oncology, Sunnybrook Health Sciences Centre, 2075 Bayview Avenue, Toronto, ON M4N 3M5; fax: 416-480-6002; andrew.loblaw@sunnybrook.ca 